\title{
الفقه المعاصر للأقليات الإسلامية
}

\section{تقرير عن المؤتمر السنوي الخامس لجمعية العلماء الاجتماعيين المسلمين في بريطانيا}

\section{21 22-20 شباط (فبراير)}

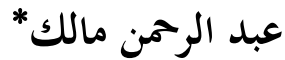

كان التعامل مع الفقه، يتسم على الدوام، بالحيوية والمرونة. وبدلاً من اعتبار الفقه مجموعة ثابتة من الأحكام القانونية، فإنه في حقيقة الأمر موضوع يشغل حياة المسلمين، ويزودهم بوسائل التعبير بلغة دينية، ذات طبيعة شخصية واجتماعية. إن فهم القيم الكامنة في الأحكام الشرعية، وكيفية استنباطها، من شأغا تمكين المجتمعات الإسلامية في البلدان الغربية من إقامة الصلة الوثيقة بين الإسلام وأوضاعهم وبيئاقم المختلفة. ولا شك في أن الوجود الإسلامي المتزايد في هذه البلدان، والمناقشات المتصاعدة حول طبيعة المواطنة والهوية، تعطي المسألة معنئ مهماً.

فهل يستطيع المسلمون في الغرب -مع إدراك التحديات والتمييز المنظم ضدهم فيه ويفقدهم الميزة والأفضلية- أن يتوصلوا إلى صيغة يعبرون فيها عن الإسلام بطريقة ترتبط بتراثهم الغني، وتكون في الوقت ذاته متناغمة مع متطلبات الحياة المعاصرة وقضاياها؟ إن المحاولات التي يبذها ويجتهد بها علماء الفقه المعاصر لتطوير فقه لخدمة الأقليات المسلمة، هي في صميم المناقشات التي بتري حالياً، حول الإسلام في أوروبا. ويشكل مشروع الفقه للأقليات الإسلامية، تحدياً عقلياً وروحياً في آن معاً.

هذه الدوافع قامت جمعية العلماء الاجتماعيين المسلمين في المملكة المتحدة، بالتعاون مع المعهد العالمي للفكر الإسلامي، والكلية الإسلامية ووكالة "Q-News" الإعلامية، بعقد مؤتمر تحت عنوان "الفقه المعاصر للأقليات المسلمة". وهو المؤتمر السنوي الخامس للجمعية، وقد عقد في جامعة وستمنستر في 
وقد أوضح الدكتور أنس الشيخ علي، رئيس اللجنة التنفيذية للجمعية في كلمته الافتتاحية، تصور المؤتمر بالنسبة لهذا الموضوع. وأعرب عن رأيه بأن فقهاً حقيقياً أصيلاً للأقليات الإسلامية، يتطلب تفاعلاً مشتركاً بين علماء الاجتماع وعلماء الشريعة، في الميدان العام الأرحب، بحيث يخرج النقاش والبحث من وراء الأبواب المغلقة. وقد وجه تحدياً للمشتركين، الذين وفدوا من أوروبا، وأمريكا الشمالية، والشرق الأوسط وجنوب شرقي آسيا، بالعمل على تطوير "آلية شاملة لفقه الأقليات"، مستمداً مبادئه من تراث الماضي، ولكن غير هياب من التحديث والتجديد.

كان ضيف المؤتمر هو الدكتور مصطفى جريك، المفتي الأكبر للبوسنة والهرسك ورئيس العلماء فيها، وهو شخصية مرموقة كانت مصدر قوة لشعب البوسنة إبان حرب البلقان. وهو يقف في موقع مميز فريد لفهم معنى أن تكون أوروبياً مسلماً، وأن تضع بتارب المسلمين الأوروبيين في إطار أوسع من الانتماء إلى أمة عالمية. وبينما أعرب الدكتور جريك عن اعتقاده بعدم الإيمان بفقه الأقليات في حد ذاته، إلا أنه أكد على ضرورة أن يفهم المسلمون أنفسهم في أوروبا بوصفهم أقلية سياسية، ولكنهم غالباً ما يكونون أكثر قدرة على القيام بواجباقم الدينية من المسلمين في سائر أرجاء العالم الأخرى. وقد رسم تصوراً للمسلمين في أوروبا يعترف بموقفهم التاريخي الفريد ومساهماهم في أوروبا؛ كما أنه وصف أوروبا بأهنا مكان للتفاعل أكثر من كونه مكاناً للمواجهة. كما أكدّ على ضرورة التفاعل المتبادل للثقافات والحاجة إلى التوازن والتسامح. وقد ختم الدكتور جريك كلمته، متحدياً المسلمين في الأطراف بأن ينظروا إلى أنفسهم كدافعين لعجلة تقدم الحضارة الإسلامية. وقد دعا أوروبا إلى جعل الإسلام مؤسسة، كما دعا مسلمي أوروبا إلى التجمع معاً ضمن منظور عالمي الآفاق.

وقد منحت جمعية العلماء الاجتماعيين المسلمين بالمملكة المتحدة جائزتا السنوية لعام 2003، وهي الجائزة المخصصة للإنجاز مدة الحياة، إلى إدوارد سعيد، تقديراً من الجمعية لمساهماته العلمية الفريدة واهتماماته الفكرية الممتدة عبر بضعة عقود. 1 وقد قدم الجائزة الدكتور الشيخ علي -بالنيابة عن اللجنة التنفيذية- إلى ميشيل عبد المسيح، وهو محام بارز وناشط فلسطيني، وكان صديقاً حميماً لإدوارد سعيد. وقد

$$
1 \text { توفي أدوارد سعيد في } 25 \text { سبتمبر } 2003 \text { بعد مرض عضال }
$$


عدد الدكتور زكي بدوي مساهمات إدوارد سعيد المهمة خخصصاً بالذكر كتابيه (تغطية الإسلام) "Covering Islam" الأكاديمية، والنظرات الاستعمارية السائدة عن الإسلام، والمسلمين والشرق الأوسط.

وقد قدمت جمعية العلماء الاجتماعيين المسلمين جائزة بناء الجسور" Building Bridges award والتفاهم بين الأديان. وقد شرحت "فرينا علم" -المحررة المسؤولة في مجلة Q-News - كتابات آرمسترونج المهمة وعرضها الموضوعي للإسلام في الكتب والمقالات، والمقابلات الإذاعية والتلفزيونية الكثيرة، وبالأخص بعد أحداث 11 أيلول. وقد عبرت الدكتورة ارمسترونج عن تأثرها البالغ وشكرها العميق لهذا التقدير الذي تعتز به، ودعت المشاركين إلى تحدي التعصب الذي يواجهونه من خلال حملة مخلصة هادفة إلى مكافحة سوء الفهم، بأسلوب خلاق. وقد استقبل المشاركون حضورها وملاحظاتها استقبالاً حاراً.

افتتحت أعمال المؤتمر ببحث قدمه الدكتور لؤي صافي، وهو رئيس جمعية العلماء الاجتماعيين المسلمين في الولايات المتحدة، وقد كانت الورقة بعنوان: "الرسالة الإبداعية للأقليات المسلمة في الغرب: التوفيق بين مفاهيم الإسلام والحداثة". وقد سبر في بحثه الآراء التي تؤمن بالدور الخلاق الذي يمكن أن يلعبه فقه الأقليات في مساعدة المسلمين على التعامل مع المفاهيم الغربية، وذلك بتطبيق الشريعة ضمن الأطر الاجتماعية المعاصرة، حيث إن تطبيق مقاصد الشريعة من شأنه أن يدعم نظاماً طبيعياً قادراً على رفع مستوى الحياة البشرية وتقدم الوضع الإنساني. وأعرب عن رأيه بأن التشريع ليس أداة من أدوات الدولة، بل هو عمل من أعمال المجتمع المدني الذي حافظ على استقلاله من تدخل الدولة، وبالتالي ضمن الحقوق القانونية المطلقة للجماعات الدينية المتعددة، وذلك خلافاً للعلمانية القائمة حالياً، والتي ترغم الأقليات على اعتماد مواقف الفئة المهيمنة. وقد أكد قوة التوسط الثقافي، ورفض مقولة الأخلاقية العالمية للعلمانية. وثم قدم الدكتور محمد الميستيري، أستاذ الفلسفة والفكر الإسلامي المعاصر في معهد العلوم الإسلامية في باريس بحثاً بعنوان: "من فقه الأقليات إلى فقه المواطنة"، وذلك بالاستفادة من بحارب المسلمين في فرنسا عندما قدم ذلك أن الفقه الكلاسيكي، في نظره، يرى الأقليات في حالة اندماج تام كأعضاء في ميثاق 
المواطنة. وفي إطار الديموقراطية، فإن الأغلبيات والأقليات هما مفاهيم مرنة. يجب أن ننتقل من مفهوم المهاجرين إلى الغرب إلى مفهوم المواطنة فيه. يجب أن يُنظر إلى فقه الأقليات من منظور هذه الحقائق المجتمعية الجديدة، وأن ترويج الإسلام في ساحة التعددية القائمة، يجب أن يبسط التعاليم الروحية مع النظرة العالمية والإنسانية. ورأى بأن العلمانية، مقبولة كأساس للتنظيم الاجتماعي، ورأى الثراء الكامن في الساحة العلمانية التي تتفاعل على ساحتها العولمة.

الدكتور زكي بدوي مدير الكلية الإسلامية في لندن تحدث بعد ذلك، فقدم بحثاً مستفيضاً تحت عنوان: "القواعد الفقهية"، التي أسماها المفاهيم التي تسيّر القانون، وهو علم منفصل يلزم إحياؤه. وانتقد الباحث الاجتهاد الصادر عن الجماعات أو اللجان، لأن اجتهاد الجماعات يصبح مهتماً بسلطتها نفسها، ولأنه يعتبر اجتهادها متفوقاً وأكثر صحة من اجتهاد الآخرين. إن الاجتهاد الصادر عن أفراد يقدمون مساهتهم ويعرضوفا للمناقشة والبحث، هو أقرب إلى روح الفقه، لأنه يرى في عملية استباط القوانين والأحكام عملية تستند إلى النقاش والقبول والتوافق. تستطيع جاليات الأقلية المسلمة أن تكون موقعاً للتفاعل العلمي الديناميكي. إن التأقلم مع تنظيمات وأطر العلمانية التي تسمح بالتعددية والحوار هو شيء ضروري، لأن ما هو أهم من أحكام الفقه نفسها هو القيم الأخلاقية التي تحمها.

أما الدكتور طه جابر العلواني -رئيس جامعة العلوم الإسلامية والاجتماعية، والرئيس الحالي لملس الفقه في أمريكا الشمالية- فلم يستطع الحضور بنفسه إلى المؤتر، لذلك بعث شريط فيديو للورقة التي أعدها بعنوان: "فقه الأقليات: ما بين الجزئيات والكليات"، والتي دعا فيه لرؤية حقائق جديدة تتعلق بجماعات الأقلية الإسلامية، والتي لا تقارن مع ما كان عليه الحال في الماضي. ويترتب على ذلك وجوب تطوير فقه الأقليات الذي يهكم أوضاع الأقليات الإسلامية، ويحفظ هويتها ويسمح بالدعوة لرفع نوعية الحياة والقيم الإنسانية عن طريق الإمكانيات التي توفرها الشريعة.

وقد تحدث الدكتور بسطامي خير، الأستاذ في جامعة بيرمنجهام، عن "الآراء القانونية (الفقهية) الإسلامية بالنسبة للأوضاع السياسية والقانونية للمسلمين في البلدان غير الإسلامية". وقد استعرض الباحث نظرية ولاية العلماء الموجودة في المدارس الحنفية والشافعية والمالكية، كوسيلة لتوفير القيادة والتوجيه القانوني 
والروحي للأقليات المسلمة. وفي إطار تاريخي استخدم هذا المبدأ للتفاوض حول درجات مختلفة من الحكم الإسلامي ومأسسته في البلدان غير الإسلامية، وهو إطار لمُ يعد ممكناً، بكل بساطة، في يومنا هذا. ومع ذلك، فإن الصورة الجلية والدقيقة للأقليات الإسلامية التي قدمها مؤرخون من أمثال المسعودي، يمكن أن تزودنا بإيحاءات يمكن تطبيقها من خلال بجالس تحكيم، أو يتم التفاوض بشأها في النهاية مع السلطات السياسية في أوروبا وغيرها. وتحدث الدكتور طاهر مهدي من جامعة فلنسيا، فركز تفكيره، مثل غيره من العلماء، على "الأقليات ومقاصد الشريعة"، وقال إنه لا يجب أن يخشى المسلمون اللجوء إلى الاجتهاد في سعيهم لوضع فتاوى جديدة لمواجهة الظروف المعاصرة. إضافة إلى ذلك، يجب علينا ألا نرى أنفسنا كمجموعة متضررة، أو أن نسمح لأنفسنا بأن نُعامل كأقلية. وبدلاً من ذلك، يجب أن نقبل حقيقة أننا مواطنون أوروبيون ينتمون إلى الدين الإسلامي. إن التشريع الإسلامي القديم هو مصدر إلهام، ولكنه ليس قابلاً للتطبيق في جميع الأحوال. هذا يعني أن علينا أن نبتعد عن التعصب. وقال أن الخطوة المهمة الأولى في إعادة الإبداع الخلاق للتشريع هي إعادة النظر في العمليات التي تميز ضد المرأة، سواء في التراث الفقهي أو الأعراف الاجتماعية.

وبينما خصص اليوم الأول لاستعراض الآفاق النظرية، خصص اليوم الثاني من المناقشات لاستعراض حقائق بحارب الأقليات المسلمة للدلالة على التحديات والفرص أمام فقه الأقليات. وقد استعرضت الدكتورة سمية بيرنيلا عويس، عضوة الأكاديمية الإسلامية السويدية، في الورقة التي قدمتها إلى المؤتمر بعنوان: "استراتيجية الزواج في أوساط الشباب المسلم في أوروبا"، الدراسات الميدانية الواسعة التي تمت في هذا الشأن، والتي تدلل على طبيعة المشاكل التي تواجه علاقات الجنسين في ظل الإطار الأوروبي. إن الشباب المسلم يعاني من نظريات تزداد تعقيداً باستمرار فيما يتعلق بمؤسسة الزواج، وتتطلب هذه المشكلة استراتيجية جديدة تركز في المقام الأول على الاختيار الشخصي، وتقلل من التركيز على إنجاب الأطفال، وقضايا العائلة الموسعة. إن زواج المرأة المسلمة من رجل غير مسلم وممارسة الفصل بين الجنسين، يجب أن تكون من أولويات البحث عند وضع مبادئ فقه الأقليات. 
وقد عالج الدكتور أحمد الخطيب، المفكر السياسي، موضوع "مسألة العلاقات الجنسية في صفوف الشباب المسلم". وقد أجرى بحثاً حول خيارات الزواج المتاحة في الموروث الفقهي، وأجرى تقييماً لميزات كل واحدة منها. وقد أبرز ثلاث خيارات متاحة: الزواج المبكر، الزواج مع نية الطلاق، والزواج المؤقت. وبالنظر إلى ما تنطوي عليه مزاولة النشاط الجنسي غير الشرعي من نتائج وخيمة بالنسبة للشباب المسلم، فإن أبحاث فقه الأقليات يمكن أن تبرر إعادة تقييم للمسلّمات التاريخية القائمة، من أجل إيجاد صيغ قانونية (فقهية) مرنة، تتيح فرص ممارسة "الحلال" للشباب. وقد ناقش المشتركون مزايا الخيارات المختلفة التي قُدمت، والنتائج السلبية الخطيرة -الاجتماعية والنفسية- التي قد تنشأ عن كل منها، ودعوا إلى بدائل ذات جذور عميقة منبثقة من روح الإسلام والقانون.

وتحدث تشارلز لو جي إيتون، المؤلف المعروف والمذيع الشهير والصوت المسموع في الإسلام البريطاني، عن فقه الأقليات في إطار من الموية المتحركة والمتنازعة. وقد دعا إلى العمل على تطوير إطار مناسب للتعبير

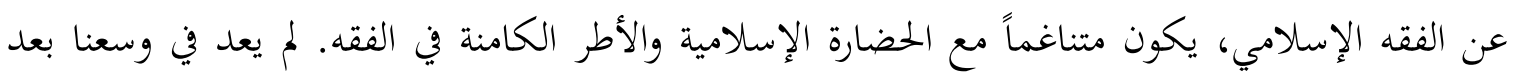
الآن أن نكون في وضع المشاكس، بل يجب أن نتخذ موقف المحاور. المرونة تمنع الثورة، وخير الأمثلة على ذلك هو ما يتم التعايش معه من إمان وحياة عادية يومية مستمدة من حقيقة الإسلام. وخلال المناقشات، لفت السيد إيتون الأنظار إلى أن النشاط الفقهي يجب أن يتم وأن يكون انعكاساً للسياق الذي يتم فيه، ولكن دونما رفض لنظام ظَل حياً وباقياً ومطوراً لقانون الشريعة على امتداد التاريخ الإسلامي. وقد حذر من أخطار النسبية، كما حذر من أن العلمانيين قد استفاقوا إلى القيم التي يمثلها الإسلام ويعتبروها تمديداً لم.

الدكتور عصمت علي، من كلية بيربك في جامعة لندن، والذي يدرّس فيها الأخلاقيات القرآنية

والنظريات القانونية فيه، قدم ورقة بعنوان: "التعددية: القوانين الإسلامية وغير الإسلامية - قضية تفسيرات"، شرح من خلالها معنى "القانون الإسلامي" في القرآن، وحاول إعادة تفسيره في ضوء الرسالة القرآنية العريضة والمشتملة على التعددية، معتبراً رسالتها بأها توسيع لنفس الرسالات العالمية التي جاءت قبلها وليس إحلالاً لمكانا. وقال إن رسالة القرآن تعبر بالدرجة الأولى عن النظريات القانونية الأساسية، وليست قانوناً وضعياً مرتبطاً بزمان ومكان. 
واستعرض دلوار حسين، وهو باحث في مؤسسة الأبحاث الإسلامية في ليستر، عملية تطبيع الإسلام في أوروبا، قائلاً إنها تختلف عن موضوع علمنة المسلمين. إن كتابة يضعها أوروبي مسلم تنطوي على تساؤلات بالغة الخطورة، حول العوامل التي تترك أثراً عميقاً في أوضاعنا. إن تأثير العولمة، ومسألة الخصوصية التي تميز إقليماً جغرافياً عن الآخر، يجب أن تحفز أجوبة مناسبة إذا كان لفقه الأقليات أن يكتسب الشرعية اللازمة. إن استخدام نظريات علم الاجتماع لحل تلك القضايا المعقدة، هو الذي يمكننا من بناء إطار قائم على فهم براجماتي ونظري لأوضاع المسلمين الأوروبين.

في الجلسة الختامية للمؤتمر، انصب اهتمام المشاركين على فحص أنماط من الفكر الشرعي الإسلامي تكون له علاقة مباشرة باهتمامات المجموعات الإسلامية التي تشكل أقليات في بلادها. أحمد تومسون وهو محام ونائب رئيس جمعية المحامين المسلمين استعرض، برصيده الواسع الذي كسبه من خلال مرافعاته المستبطة من القانون (الفقه) الإسلامي أمام المحاكم، ورقة بحث قدمها تحت عنوان: "استيعاب القانون الإسلامي للأحوال الشخصية ضمن القانون المحلي للمملكة المتحدة". وقال إن قانون الأحوال الشخصية هو أحد أكثر قوانين الشريعة حيوية وديمومة. وبالنظر إلى أن الأقليات المسلمة تسعى باستمرار إلى حل قضاياها ونزاعاتما العائلية على أسس من قانون الأحوال الشخصية الراسخ، فإن هنالك إمكانية لدبجه في القانون الإنجليزي المتوارث. وقد أعرب تومسون عن وجهة نظره بأن نمو وانتشار استخدامات هذا القانون سيجعلها متجذرة ومستندة إلى الأسبقيات القانونية المتعامل بها، مستخدمين في ذلك وسيلة التحكيم الملزم. مثل هذه الطرق القانونية القائمة يمكن، في فاية الأمر، أن تتطور إلى نظام للتحكيم من قبل قضاة يتمتعون بكفاءات عالية وثقة الذين يوكلون أمرهم إليهم.

وقد أعرب الدكتور إحسان يلمز عن تخوفه من نشأة ما يسمى "بمجتهدي التجزئة" و "فقه الأقليات"، وبالتالي تمزق وتفرق بجمل النظام الشرعي الإسلامي عندما يمارس الأفراد (التخير) بين المدارس الفكرية والمذهبية. ومع الإدراك بأن القوانين، وما ينبثق عنها من تفرعات وتفسيرات، هي من شؤون المجتمعات المدنية، فإن هذا يعني بأن الفوضى القانونية المحتملة يجب أن تعالج وتدبر أيضاً. ويرى الدكتور يالمز أن 
الحركات القائمة على الإيمان تعطي شرعية للأحكام المنبثقة عن اجتهاد اللجان، بحيث تعطي رواجاً وقبولاً لنظريات قانونية مبتكرة وتجعل عملية الاجتهاد عملية قابلة للتطبيق.

وفي ملاحظاته المتامية، شكر الدكتور أنس الشيخ علي المتحدثين والمشاركين، مؤكداً أن هدف جمعية العلماء الاجتماعيين المسلمين هو الجمع بين النظرية وبين التطبيق العملي. وذكر أنه، وكنتيجة لأعمال المؤتمر السنوي الرابع حول تعليم المسلمين في أوروبا، فقد وضعت جمعية العلماء الاجتماعيين المسلمين ورقة عمل عنواها "المسلمون والتعليم"، تبحث الورقة في قضايا واسعة ومتشعبة في حقل التعليم في المملكة المتحدة، على أمل أن تشكل أساساً للحوار بين دائرة التعليم والمهارات من جهة، وبين مثثلي الجاليات الإسلامية؛ وسوف تنشر هذه الدراسة في غاية شهر آذار عام 2004. وأضاف قائلاً أنه بعد المناقشات التي جرت خلال انعقاد المؤتمر السنوي الخامس، فسوف تنظر جمعية العلماء الاجتماعيين المسلمين، خلال اجتماعها القادم، في إمكانية تفويض علماء اجتماع بوضع أبحاث محددة؛ سوف تركز تلك الأبحاث، ليس فقط على القضايا المعاصرة التي تواجه الجاليات الإسلامية في أوروبا، ولكن على قضايا وتحديات أخرى قد تنشأ في المستقبل. والمؤمل أن توضع مثل هذه الدراسات أمام مجالس الفقه وأمام الفقهاء الأفراد للنظر فيها وتقييمها. 